\title{
O Ser e o Trabalho: um estudo de avaliação de fatores indicativos de satisfação
}

\begin{abstract}
Samuel Rodrigues Lima ${ }^{\mathrm{a}}$, Sefisa Quixadá Bezerra ${ }^{\mathrm{b}}$, Levi Leonido ${ }^{\mathrm{c}}$, Mário Cardoso ${ }^{\mathrm{d}}$, Elsa Morgado ${ }^{\mathrm{e}}$

${ }^{\text {a } U n i v e r s i d a d e ~ E s t a t u a l ~ d o ~ V a l e ~ d o ~ A c a r a u ́, ~ S o b r a l, ~ B r a s i l, ~ s a m u l i m a 4 @ ~ g m a i l . c o m, ~}{ }^{\text {b Universidade }}$ Estatual do Vale do Acaraú, Sobral, Brasil, sefisaquixada@gmail.com, Universidade de Trás-osMontes e Alto Douro, Vila Real, Portugal, Universiade Católica Portuguesa (CITAR), Porto, Portugal, levileon@utad.pt , Instituto Politécnico de Bragança, Bragança, Portugal, cardoso@ipb.pt, Universidade Católica Portuguesa - Centro de Estudos Filosóficos e Humanísticos da Universidade Católica Portuguesa - Braga, UTAD, IPB, Portugal, levielsa@utad.pt.
\end{abstract}

\section{$\underline{\text { Resumo }}$}

A satisfação com o trabalho possui importante relevância para o bem-estar dos colaboradores e consequentemente proporciona melhor desempenho em suas atividades. Alguns autores consideram que para se ter noção adequada de como mensurá-la deve-se analisar a relação do colaborador com alguns fatores, dentre os quais sempre são estudados pela literatura da área e por isso escolhidos para compor esse trabalho: a natureza e condições do trabalho, salário, sistema de promoções de cargo, equipe de trabalho e o superior imediato. Neste sentido, o presente estudo buscou avaliar o nível em que os referidos fatores indicativos de satisfação estão impactando no trabalho de colaboradores de uma rede de padarias em um município do Estado do Ceará - Brasil. Para tanto, realizou-se uma pesquisa exploratória descritiva, referenciada com levantamento de dados em campo. Os principais autores pesquisados foram Sarathy e Barbosa (1981), Tamayo (1998), Bergamini (2000), Chiavenato (2002, 2003) e Martinez e Paraguay (2003). Os resultados demonstraram que quanto às dimensões natureza do trabalho, superior imediato e equipe de trabalho, no local pesquisado, a avaliação foi muito positiva, enquanto as dimensões ascensão de cargo $e$ remuneração demonstraram uma média satisfação e, havendo neles a possibilidade de avaliação por parte da empresa. Os resultados das dimensões são coerentes com os dados encontrados na avaliação geral com os indicadores de qualidade de vida.

Palavras-chave: Comportamento organizacional, satisfação no trabalho, gestão de pessoas. 


\begin{abstract}
The satisfaction with the work has important relevance for the well-being of the employees and consequently provides better performance in its activities. Some authors consider that in order to have adequate understanding of how to measure it, one should analyze the relation of the collaborator to some factors, among which are always studied by the literature of the area and therefore chosen to compose this work: the nature and conditions of the work, salary, job promotion system, work team and immediate superior. In this sense, the present study sought to evaluate the level at which these factors indicative of satisfaction are impacting the work of employees of a bakery network in a municipality of the State of Ceará - Brazil. For that, a descriptive exploratory research was carried out, referenced with data collection in the field. The main authors searched were Sarathy e Barbosa (1981), Tamayo (1998), Bergamini (2000), Chiavenato (2002, 2003), and Martinez and Paraguay (2003). The results showed that, in terms of the nature of the work, the immediate superior and the work team, in the local searched, the evaluation was very positive, while the ascension of positions and remuneration showed an average satisfaction and, having in them the possibility of evaluation by the company. The results of the dimensions are consistent with the data found in the general evaluation with the indicators of quality of life.
\end{abstract}

Keywords: Organizational behavior, job satisfaction, people management.

\title{
1. Introdução
}

Nos tempos que decorrem, o ser humano passa a maior parte de seu tempo em organizações, onde, de acordo com Chiavenato (2002), o ambiente caracteriza-se por condições físicas e materiais e também psicológicas e sociais. Esta afirmação reforça a compreensão da importância de perceber o comportamento do ser humano nas organizações, em especial os aspetos relacionados a satisfação do colaborador em as suas responsabilidades no trabalho (Marqueze \& Castro Moreno, 2005). Neste sentido, este trabalho teve como objetivo, avaliar o nível em que os alguns fatores indicativos de satisfação interferem no trabalho. Foram utilizados os fatores definidos por Tamayo (1998) e Sarathy e Barbosa (1981) como referência para o estudo em campo, nomeadamente: satisfação com o salário, colegas de trabalho, superior imediato, ascensão de cargo e trabalho em si, e também o modelo de qualidade de vida proposto por Walton (1979 apud 
Alves, 2010, p. 7), tendo como objeto de estudo uma empresa no segmento de panificação na cidade de Sobral, Estado do Ceará- Brasil.

\section{Enquadramento}

No campo da Teoria Geral da Administração, o comportamento humano nas organizações tem sido estudado desde a escola de Administração Científica, fundada por Frederick W. Taylor, que procurou conhecer formas de maximizar a produção industrial no menor tempo, ignorando a essência humana e social, definindo que a única fonte motivacional do trabalhador centrava-se na concessão do salário, criando a definição de "homo economicus" (Chiavenato, 2003). Tentando corrigir a forte tendência de desumanização no trabalho defendida pelo pensamento taylorista, o psicólogo Elton Mayo desenvolveu a Teoria das Relações Humanas, definindo o "homem social" ao reconhecer que os trabalhadores possuem sentimentos, temores, desejos e singularidades sociais que são motivadas por certas necessidades, e só alcançam sua satisfação através dos grupos com os quais interagem. (Carvalho, 2002). Após os estudos de Taylor e Mayo foram desenvolvidas diversas teorias que reforçaram a ênfase dada às tarefas e às pessoas, analisando os impactos da estrutura, do ambiente e da tecnologia para as organizações. Com esta evolução teórica nasceram novas conceções sobre o ser humano, tais como Homem Organizacional e Administrativo durante a Teoria Neoclássica, Homem Organizacional nas Teorias da Burocracia e Estruturalista, Homem Administrativo na Teoria Comportamental, o Homem Funcional na Teoria de Sistema e o Homem Complexo na conceção da Teoria da Contingência (Chiavenato, 2003).

\subsection{O Trabalho dignifica o homem. O Trabalho significa para o homem}

O trabalho abrange grande parte da vida do ser humano e pode ser visto como fonte de significado pessoal, reconhecimento, gratificação económica, meios de conseguir autorrealização e autodesenvolvimento (Pereira, 2006). Martinez e Paraguay (2003) afirmaram que o trabalho é uma das melhores formas de o homem expressar a sua singularidade, algo que o completa e dá sentido a vida, por este motivo, é visto como uma das fontes da felicidade humana, onde a felicidade no trabalho é alcançada através da satisfação plena e necessidades biopsicossociais, da sensação de bem-estar e do sentido de utilidade no exercício de suas responsabilidades no trabalho. De acordo com Frankl (2003), o homem só se torna homem e só é completamente ele mesmo quando fica absorvido pela dedicação a uma tarefa, quando se esquece a si mesmo no serviço a uma causa ou no amor a uma pessoa. Em trabalhos desenvolvidos sobre a Psicodinâmica do trabalho, Dejours e 
Abdoucheli (1994) extraíram aquilo que era potencialmente desestabilizador para a saúde mental dos trabalhadores, identificaram que todas as pressões aparecem como decorrentes da organização do trabalho.

\subsection{Satisfação no trabalho}

De acordo com Martinez e Paraguay (2003, p. 61) “A satisfação no trabalho é um fenômeno amplamente estudado, e esse interesse decorre devido à influência que ela pode exercer sobre o trabalhador, afetando sua saúde física e mental, atitudes, comportamento profissional, social, tanto com repercussões para a vida pessoal e familiar do indivíduo como para as organizações". Spector $(2003$, p. 241) refere que "a satisfação deve resultar do desempenho", ou seja “o desempenho deve resultar da satisfação". Pereira (2006, p. 20) salienta que "a satisfação no trabalho é o resultado dos sentimentos positivos e negativos que uma pessoa apresenta em relação ao seu trabalho, ou seja, é uma resposta emocional determinada pela interação das tarefas desempenhadas, das condições físicas e sociais do local de trabalho". Moretti (2010, p. 15) descreve que "é estudada como uma das mais importantes variáveis da área de comportamento organizacional". Outro assunto relacionado com a satisfação e motivação que também congrega para o bom desempenho do colaborador é Qualidade de Vida Extensiva ao Trabalho ou a conhecida QVT. A QVT é uma ferramenta indispensável para perceber a importância dos funcionários. Os seus programas têm o objetivo de proporcionar espaços de trabalhos mais agradáveis, condições melhores nos aspetos: saúde, físico, emocional, social, além de buscar preparar as equipes, integrar os setores com seus superiores, entre outras vantagens que melhorem o nível de satisfação dos envolvidos com a instituição (Ribeiro, 1993). Alves (2010) relata que inúmeros instrumentos para avaliar a QVT têm sido criados e utilizados, sendo que parte deles apresentam a satisfação profissional como definição principal.

\subsection{A Importância das dimensões da satisfação no trabalho}

Considerando que a satisfação do profissional com o trabalho é um estado emocional advindo da interação com outros profissionais, suas peculiaridades pessoais, valores e expectativas com o ambiente e a organização do trabalho torna-se necessário para o empregado estar satisfeito com o trabalho. Assim como estar igualmente satisfeito com o trabalho em si, com o seu superior imediato. Pelo que as relações interpessoais devem ser positivas no ambiente profissional (fatores intrínsecos), com uma remuneração salarial digna e seguida com políticas de promoção e ascensão justas (fatores extrínsecos). Este ajustamento pode ser considerado como normal, quando as necessidades humanas são satisfeitas dentro das exigências impostas pela própria natureza humana. Havendo 
descompasso entre os objetivos individuais e organizacionais ocorrerá o conflito e, consequentemente, ao estresse, que quando enfrentado adequadamente, representa um terreno fértil ao desajustamento individual (Bergamini, 2010).

\section{METODOLOGIA}

A investigação caracteriza-se como qualitativa, tendo como método o estudo de caso. A interpretação dos dados por meio destes softwares SPSS versão 22.0 e Excel 2010, permitiu obter conhecimentos sobre o perfil dos participantes, nível de satisfação em relação às dimensões estudadas e avaliar o nível de QVT geral da organização. O universo desta pesquisa englobou os colaboradores das duas unidades de uma empresa do setor de panificação em Sobral, sendo composto por 59 colaboradores com contrato formal de trabalho. A amostra correspondeu a 49 servidores, correspondendo a $83 \%$ da população, tendo caráter do tipo não probabilístico por aceite.

$\mathrm{Na}$ recolha de dados usou-se um questionário dividido em 3 partes: (1) Pesquisa sociodemográfica; (2) pesquisa com afirmativas sobre fatores de satisfação no trabalho; e (3) pesquisa de avaliação dos indicadores de Qualidade de Vida no Trabalho. Os dados principais foram obtidos através de questionário adaptado de um instrumento desenvolvido por Sarathy e Barbosa (1981), apresentado como Escalas para Medir Satisfação com o Emprego - EMSE, adicionado de complementos para análise do perfil dos colaboradores e de um questionário adaptado pelos autores desse estudo baseado em seis dos oito indicadores de QVT propostos por Walton (1974, apud Alves, 2010, p. 79). No total o instrumento possui 78 questões (6 perguntas para análise do perfil do entrevistado, 66 questões com afirmativas da EMSE e 6 afirmativas sobre os indicadores de QVT). Para avaliar as afirmativas sobre satisfação e QVT optou-se pelo uso da escala Likert tendo em vista que o modo mais comum de se aferir a satisfação no trabalho se dá por meio de escalas deste tipo. Foram utilizadas as seguintes variáveis: ordinal, para as afirmativas das escalas de satisfação com o emprego, e com os indicadores de QVT, tipo Likert. Nas afirmativas com as variáveis intrínsecas e nos indicadores de QVT variou de 1 a 5 (de discordo totalmente a concordo totalmente) e de 1 a 3 nas variáveis extrínsecas (tendo o mesmo parâmetro, porém sendo o número 2 a opção "nem concordo nem discordo"); variável nominal (sexo, estado civil e posição ocupada na instituição), variável numérica intervalar (tempo de trabalho na instituição, faixa etária e salarial mensal). O instrumento foi aplicado in loco pelos pesquisadores. 


\section{Análise dos resultados}

Análise do perfil dos respondentes: $51 \%$ dos entrevistados são homens e $49 \%$ mulheres. A faixa etária a maioria dos colaboradores, $43 \%$, tem entre 21 e 29 anos e a segunda está entre 30 a 34 anos (24,5\%). A maior parte são solteiros (59\% do total). Em relação à faixa salarial mensal, constatou-se que todos os colaboradores recebem de 1 a 2 salários mínimos. Ao que tange a posição ocupada na empresa (88\% está no nível operacional e $12 \%$ em nível tático especializado). Nenhum colaborador do nível estratégico respondeu. Quanto ao tempo de serviço na organização, constatou-se que a maioria dos servidores, com 55\%, está na organização há menos de 1 ano. Em seguida, aparecem os funcionários que possuem de 2 a 4 anos e 11 meses no ambiente (24,5\%).

\section{Análise de cada dimensão:}

Satisfação com a natureza do trabalho: a média 4,22 obtida das 12 afirmativas de satisfação, e pela média de 2,66 das 5 de insatisfação, onde a última caracteriza que os colaboradores discordam em partes que determinadas condições ocorram, levando a deduzir que neste caso há equilíbrio nos polos satisfação e insatisfação. Os índices empregados como medidas para conhecimento com esta dimensão de satisfação foram relacionados aos fatores respeito, onde a primeira refere-se ao cargo e atividades realizadas para a organização e a segunda para as condições humanas e de identidade pessoal. Houve destaque para a afirmativa que aquele trabalho era de natureza "exaustiva". Com as menores médias nas afirmativas de insatisfação, ficaram que "o trabalho era frustrante e enfadonho", sugerindo coerência com as médias positivas relacionadas as condições humanas e de identidade, ou seja, os maiores índices foram de "encantamento, satisfação, prazer, uso de criatividade e realização pessoal e profissional" no ambiente de trabalho e com as atividades realizadas.

Satisfação com o superior imediato: neste índice os servidores demonstram ampla satisfação com o seu superior representado pela média 4,39, a maior média de satisfação por dimensões da pesquisa, considerando-se as 13 afirmativas de satisfação. Com pontuação 1,52, discordaram quase que totalmente das afirmativas que sugerem insatisfação. Nesta dimensão as afirmativas são: que os superiores expressam respeito e buscam proporcionar bem-estar aos colaboradores (coerente com a dimensão anterior), mostrou influência do líder na organização, repassam informações às suas equipes, demonstram a inteligência e tratamento igualitário, sem favoritismos.

Satisfação com a equipe de trabalho: a média das 12 afirmativas que demonstraram insatisfação são de 2,16. As 5 afirmativas que sugerem satisfação tiveram pontuação acima de 4, tendo como média geral 4,27. Destaque entre as afirmativas com teor de insatisfação, 
o índice "intrometem-se na minha vida", obtendo a menor média, 1,45. Dois índices com médias acima de 2,5 chamaram a atenção: "membros da equipe insatisfeitos" e "conversam muito durante o trabalho". Mesmo que ambas estejam no nível de discordar em partes, indica que a prática de conversar muito durante o expediente existe. Neste caso deve-se recordar que isso pode expressar àquele indicativo, já apresentado, como um início de ataque contra a empresa, como mencionado por Ribeiro (1993, p.38) ao listar possíveis ações de um funcionário insatisfeito.

Satisfação com a possibilidade de promoção/ascensão de cargo: na presente e na última dimensão de satisfação no trabalho, a quantidade de afirmativas para análise é menor que nas dimensões anteriores, bem como é reduzida a escala Likert de 5 para 3 alternativas. Isso deve-se por conta destas dimensões representarem variáveis extrínsecas ao colaborador que requerem respostas objetivas do tipo sim, não sei e não. Com média 2,43, a maioria dos colaboradores afirmam que na organização existem oportunidades de promoção. Com média 1,53, de teor insatisfatório, os respondentes defendem que as políticas de ascensão da empresa não são injustas.

Satisfação com a remuneração: obteve média 2,07, este dado aponta que os funcionários não concordam nem discordam em estarem satisfeitos com seu salário. A média mencionada, de insatisfação pontuou em 1,77. Num contexto abrangendo as dimensões de satisfação com pontuações pouco acima de 2 , os respondentes não concordam nem discordaram quanto aos índices: salário suficiente para despesas básicas como alimentação e contas da casa, proporciona uma vida confortável, salário bom e salário justo para o cargo. Destaque para o item que questionou se os respondentes consideram que a empresa distribui os seus lucros de forma justa, que foi a única afirmativa de satisfação a ficar abaixo do nível 2, indicando discordância parcial com média 1,69. Esta dimensão apresentou-se como a de menor nível de satisfação aos colaboradores.

QVT: os resultados das dimensões de satisfação, foram analisados a partir do Gráfico 1, onde constam 6 das 8 dimensões propostas pelo modelo de Walton para avaliar o nível de QVT, comparando com os resultados das dimensões de satisfação estudados. O termo constitucionalismo está ligado a existência de regras, normas de convívio no ambiente. Este foi o indicativo que mais se destacou na pesquisa, obtendo média 4,53. Em termos de coerência com os dados analisados nas dimensões de satisfação pode-se comprovar que nas 3 dimensões de fatores intrínsecos esta característica foi a que mais se destacou. Quanto ao segundo indicador com melhor avaliação destaca-se, as condições de trabalho adequadas com média 4,29, Integração social no trabalho foi o terceiro indicador mais bem avaliado com média 4,16 . 
Gráfico 1. Médias dos indicadores de Qualidade de Vida no Trabalho

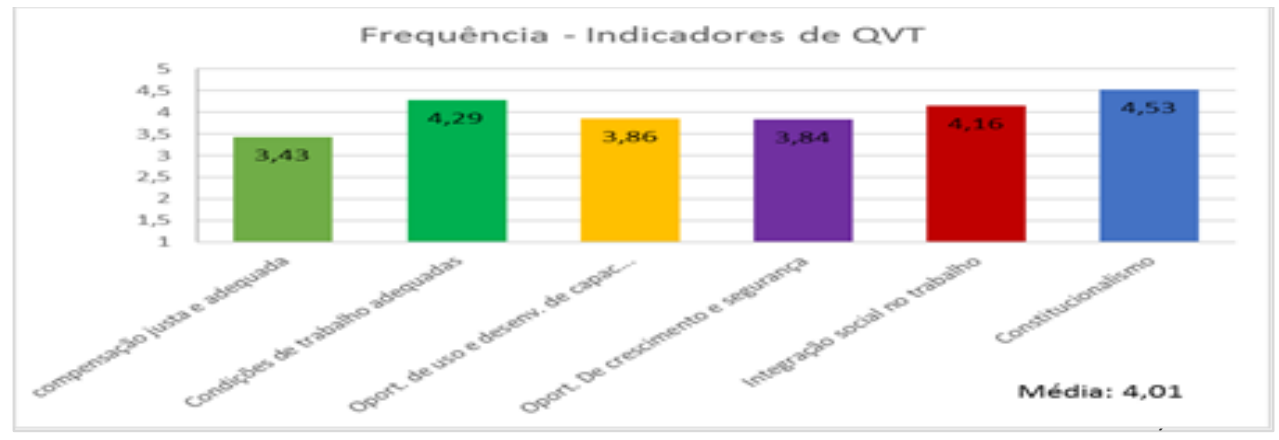

\section{Conclusões}

Numa análise geral, os participantes mostraram-se satisfeitos com as condições que caracterizam o seu trabalho, o que pode significar que estejam designados nas áreas e setores baseados nas suas habilidades e conhecimentos, além de estarem num ambiente adequado ao seu bem-estar. Também se mostraram satisfeitos com o seu superior imediato e equipe de trabalho. Esta satisfação pode ser vinculada ao relacionamento interpessoal, já que a ênfase maior se deu nas afirmativas que indicam respeito. Pode-se inferir que a instituição possui um bom ecossistema de trabalho. Foi possível identificar uma certa indiferença em relação às afirmativas de ascensão na organização. Essa consideração pode ser resultado da grande quantidade de funcionários com menos de 1 ano na organização, equivalendo a $55 \%$ dos entrevistados, o que sugere pouco conhecimento sobre esta dimensão. Na última dimensão, a escala de remuneração recebeu a menor média, porém figurando na alternativa que equivale a "não concordo e nem discordo". Neste caso, sugerese que, diante destas condições, a empresa reveja o seu plano de remunerações, observando detalhes como esforço físico, periculosidade, responsabilidade auferida aos cargos de trabalho, entre outros, e que realize ajustes, se necessários. Comparando-se todas as médias e algumas afirmativas dispostas nas dimensões estudadas com os índices de QVT, concluiuse que há coerência no cruzamento de dados, indicando satisfação com a maior parte das condições.

\section{Referências}

Alves, E. F. (2010). Qualidade de vida no trabalho: indicadores e instrumentos de medidas. Diálogos \& Saberes, 6(1), 77-87. 
Bergamini, C. W. (2010). Psicologia aplicada à administração de empresas: psicologia do comportamento organizacional. São Paulo: Atlas.

Carvalho, T. M. X. B. (2002). O homem nas organizações. Revista Centro de Ciências Administrativas, 8(1), 32-37.

Chiavenato, I. (2002). Recursos Humanos. São Paulo: Atlas.

Chiavenato, I. (2003). Introdução à teoria geral da administração: uma visão abrangente da moderna administração das organizações. Rio de Janeiro: Elsevier.

Dejours, C., \& Abdoucheli, E. (1994). Itinerário teórico em psicopatologia do trabalho. Psicodinâmica do trabalho: contribuições da escola dejouriana à análise da relação prazer, sofrimento e trabalho. São Paulo: Atlas.

Frankl, V. (2003). Sede de sentido. São Paulo: Quadrante.

Marqueze, E. C., \& Castro Moreno, C. R. (2005). Satisfação no trabalho - uma breve revisão. Revista Brasileira de Saúde Ocupacional, 30(112), pp. 69-79

Martinez, M. C, \& Paraguay, A. I. B. B. (2003). Satisfação e saúde no trabalho: aspectos conceituais e metodológicos. Cadernos de psicologia social do trabalho, 6, 59-78.

Moretti, G. J. S. (2010). O perfil e a satisfação no trabalho dos professores dos cursos de graduação em administração das instituições de Cursos superiores Privadas da cidade de Ribeirão Preto $(S P)$. (Dissertação de Mestrado). Universidade de São Paulo, Brasil.

Pereira, J. P. E. (2006). A satisfação no trabalho: uma aplicação ao sector hoteleiro da Ilha de São Miguel. (Dissertação de Mestrado). Universidade dos Açores, Portugal.

Ribeiro, C. R. M. (1993). A Empresa Holística. Petrópolis, RJ: Vozes.

Sarathy, R., \& Barbosa, J. D. (1981). Fatores explicativos da permanência e saída do emprego. Revista de Administração de Empresas, 21(1), 17-29.

Spector, P. E. (2003). Psicologia nas organizações. São Paulo: Saraiva.

Tamayo, A. (1998). Valores organizacionais: sua relação com satisfação no trabalho, cidadania organizacional e comprometimento afetivo. Revista de Administração, 33(3), 56-63. 